\title{
Article \\ On Cohomology of Simple Modules for Modular Classical Lie Algebras
}

\author{
Sherali S. Ibraev*(D), Larissa S. Kainbaeva (D) and Saulesh K. Menlikozhaeva (D)
}

Department of Physics and Mathematics, Institute of Natural Science, Korkyt Ata Kyzylorda University, Aiteke bie St. 29A, Kyzylorda 120014, Kazakhstan; larissa_kain@mail.ru (L.S.K.); saulesh_menli@mail.ru (S.K.M.)

* Correspondence: ibrayevsheraly@gmail.com

check for updates

Citation: Ibraev, S.S.; Kainbaeva, L.S.; Menlikozhaeva, S.K. On Cohomology of Simple Modules for Modular Classical Lie Algebras. Axioms 2022, 11, 78. https://doi.org/10.3390/ axioms11020078

Academic Editor: Sergey V. Ludkovsky

Received: 23 December 2021 Accepted: 10 February 2022 Published: 16 February 2022

Publisher's Note: MDPI stays neutral with regard to jurisdictional claims in published maps and institutional affiliations.

Copyright: (c) 2022 by the authors. Licensee MDPI, Basel, Switzerland. This article is an open access article distributed under the terms and conditions of the Creative Commons Attribution (CC BY) license (https:// creativecommons.org/licenses/by/ $4.0 /)$.

\begin{abstract}
In this article, we obtain some cohomology of classical Lie algebras over an algebraically closed field of characteristic $p>h$, where $h$ is a Coxeter number, with coefficients in simple modules. We assume that these classical Lie algebras are Lie algebras of semisimple and simply connected algebraic groups. To describe the cohomology of simple modules, we will use the properties of the connections between ordinary and restricted cohomology of restricted Lie algebras.
\end{abstract}

Keywords: Lie algebra; algebraic group; simple module; cohomology

\section{Introduction}

The Lie algebra cohomology was first introduced in 1929 by Cartan for a one-dimensional trivial module in order to extend de Rham's cohomological methods for Lie algebras [1]. It was generalized for arbitrary modules by Chevalley and Eilenberg in 1948 [2]. Restricted cohomology for restricted Lie algebras with coefficients in restricted modules was introduced by Hochschild in 1954 [3].

Lie algebras and their cohomology theories are widely used in mathematics and physics. The methods of the cohomology theory of Lie algebras is used to study the cohomology of principal bundles and homogeneous spaces [4]. Central extensions and deformations of Lie algebras, used in various branches of physics, are of cohomological origin. For example, the Heisenberg Lie algebra, which arises in the description of onedimensional quantum mechanical systems, is a central extension of a two-dimensional Lie algebra [5,6]. The Virasoro algebra, widely used in two-dimensional conformal field theory and in string theory, is a central extension of the Witt algebra [7]. Deformations of static kinematical Lie algebras obtained in [8] were used to classify kinematic superpaces in [9]. The tools of the cohomological theory of Lie algebras are also applied to construct a quantum model of the universe [10] and to study electromagnetic fields [11].

Cohomology theory is also being developed for $n$-Lie algebras, Lie super-algebras and some other non-associative algebras. In [12], the authors define the representation of $n$-LieDer pairs and study their cohomology and, in particular, describe the central extensions of $n$-LieDer pairs as the first cohomology of the trivial module. The Betti numbers of complex nilpotent Lie superalgebras of dimension $\leq 5$ were calculated in [13]. The cohomology of non-associative algebras with metagroup relations and their applications are studied in [14-17].

For many classes of Lie algebras in characteristic zero, their properties are well studied. In the case of a positive characteristic, the situation is different. Some properties of Lie algebras of characteristic zero for their modular analogs are not preserved. For example, in positive characteristic, there exist finite-dimensional Lie algebras that admit non-trivial deformations, while the corresponding Lie algebras in characteristic zero are rigid objects. This fact complicates the classification of finite-dimensional simple Lie algebras, and the classification of finite-dimensional simple Lie algebras in positive characteristic has not yet been completed. In the cohomological theory of Lie algebras in positive characteristic, many 
facts also keenly differ from the case of characteristic zero. For example, the cohomology of classical Lie algebras over the field of complex numbers is completely described. They are non-trivial only for trivial modules. In the modular case, there are non-trivial modules with non-trivial cohomology. Nowadays, the cohomology of non-trivial modules of classical Lie algebras over a field of positive characteristic has been intensively studied. Nevertheless, there are very few known facts about the cohomology of simple modules.

Consider a Lie algebra $\mathfrak{g}$ of classical type over an algebraically closed field $k$ of characteristic $p>0$. Let $G$ be a semisimple, simply connected algebraic group with the Lie algebra $\mathfrak{g}$ and $G_{1}$ be the first Frobenius kernel of $G$. It is well known that the representation theory of $G_{1}$ coincides with the restricted representation theory of $\mathfrak{g}$. Then, in the restricted region, a rational $G_{1}$-module can be identified with a module of the restricted universal enveloping algebra $\mathfrak{u}(\mathfrak{g})$. Hence, for the restricted $\mathfrak{g}$-module $V$, the restricted cohomology $H_{*}^{n}(\mathfrak{g}, \mathrm{V})$ coincides with the cohomology $H^{n}\left(G_{1}, V\right)$. This fact and the spectral sequence obtained in $[18,19]$ allow us to obtain some useful information about the properties of the usual cohomology $H^{n}(\mathfrak{g}, V)$.

In particular, the description of the cohomology of simple modules is one of the interesting problems in the cohomology theory of classical modular Lie algebras. In the case where $V$ is a simple module, the cohomology $H^{n}(\mathfrak{g}, V)$ is studied in detail for $n=1$ [20] and described for classical Lie algebras of small dimensions: in [21] for $\mathfrak{s l}_{2}(k)$; in [22,23] for $\mathfrak{s l}_{3}(k)$ and $p>3$; in [24] for $\mathfrak{s l}_{3}(k)$ and $p=2$; in [25] for $\mathfrak{s l}_{3}(k)$ and $p=3$. The spaces of outer derivations and local deformations of classical simple Lie algebras are also known as the first and second cohomology of the adjoint module, respectively [26-28].

In $[29,30]$, the structure of some Weyl modules was described. This means that the formal characters of the associated simple modules are known as well. The cohomology of these simple modules for $G$ has been described $[29,30]$. However, the cohomology for the Lie algebra $\mathfrak{g}$ with coefficients in these simple modules is still unknown, except for the cohomology of the first and second degrees. In the case of first cohomology, all three cohomology, i.e., the cohomology for $\mathfrak{g}, G$ and $G_{1}$ are pairwise isomorphic [31]. The second cohomology are also pairwise isomorphic, except in the case where $\mathfrak{g}$ is the Lie algebra of type $B_{2}$ [32].

The known facts obtained in the cohomology theory of algebraic groups in positive characteristic and their Lie algebras stimulate the study of the following problems in the restricted region:

- examine the cohomology of simple modules for $\mathfrak{g}$;

- examine the cohomology of simple modules for $G_{1}$;

- determine the connections between the cohomology of simple modules for $\mathfrak{g}, G_{1}$, and $G$.

In this paper, we study the first of these problems for the simple modules described in $[29,30]$. We have calculated the cohomology of classical Lie algebras over an algebraically closed field of characteristic $p>h$, where $h$ is a Coxeter number, with coefficients in simple modules whose highest weights belong to alcoves along the walls of the dominant Weyl chambers and close to them alcoves. These results can be used to describe the cohomology of simple modules for reductive Lie algebras and Lie algebras of Cartan type. For example, the calculation of the cohomology $H^{i}\left(\mathfrak{g l}_{n}(k), V\right)$ can easily be reduced to the calculation of the cohomology $H^{j}\left(\mathfrak{s l}_{n}(k), V\right)$ for some $j \leq i$. In the case of Lie algebras of Cartan type, as is known, their cohomology with coefficients in simple modules depends on the cohomology of the classical components in their natural grading. For cohomology of small degrees, some such applications can be found in $[24,33]$. Our results allow us to obtain similar results for cohomology of higher degrees.

The description of the cohomology of classical Lie algebras over an algebraically closed field of characteristic zero with coefficients in a trivial one-dimensional module is known [34] (pp. 173-174). Since in the case where $p>h$, the killing form on $\mathfrak{g}$ is nondegenerate, the cohomology results for one-dimensional trivial module in characteristic zero remain valid also for positive characteristics of an algebraically closed field. With these 
facts in mind, we will formulate the main part of our results in terms of cohomology with coefficients in one-dimensional trivial module. We denote by $H^{n}(\mathfrak{g})$ the $n$-cohomology of $\mathfrak{g}$ with coefficients in $k$.

The article is organized as follows. In Section 2, we provide preliminary information. Section 2.1 is devoted to the main notation and some concepts used in the article. Section 2.2 contains the necessary known facts to prove the main results. Here, we also give the algorithm for calculating the cohomology of simple modules based on these known results. Although this algorithm as a tool of homological algebra is not new, it allows us to obtain new results for the cohomology of simple modules. In Section 3, we state the main results of this paper and present their proof. Theorem 1, given in Section 3.1, is devoted to the cohomology of classical Lie algebras with coefficients in simple modules, isomorphic to quotient modules of Weyl modules with a simple radical by the maximal submodules. Theorem 2 describes the cohomology of classical Lie algebras with coefficients in some simple modules, which are isomorphic to quotient modules of Weyl modules with a nonsimple radical by the maximal submodules. Section 3.2 is devoted to the proofs of Theorems 1 and 2. A summary of the cohomology $H^{n}(\mathfrak{g})$ is given in Appendix A.

\section{Preliminaries and Calculation Method}

\subsection{Preliminaries}

Let us keep the notation introduced in the introduction. We denote the rank of $\mathfrak{g}$ by $l$. Let $R$ be a root system of $\mathfrak{g}$ and assume that $R \subset \mathbb{R}^{m}$, where $\mathbb{R}$ is the set of real numbers and

$$
m=\left\{\begin{array}{c}
l+1 \text { if } g=A_{l} ; \\
l \text { if } g=B_{l}, C_{l}, D_{l} ; \\
8 \text { if } g=E_{6}, E_{7}, E_{8} ; \\
3 \text { if } g=G_{2} \\
4 \text { if } g=F_{4} .
\end{array}\right.
$$

On $\mathbb{R}^{m}$, there is the usual euclidian inner product $($, ). This leads to the natural pairing $\langle\rangle:, \mathbb{R}^{m} \times \mathbb{R}^{m} \rightarrow \mathbb{R}$ given by $\langle\lambda, \mu\rangle=\left(\lambda, \mu^{v}\right)$, where $\mu^{v}=\frac{2}{(\mu, \mu)} \mu$. Let $R^{+}$be the set of positive roots and $\Delta=\left\{\alpha_{1}, \alpha_{2}, \cdots, \alpha_{l}\right\}$ be the set of simple roots.

Let $T \subset G$ be the maximal torus and $B$ be the Borel subgroup corresponding to the negative roots. We denote by $U$ the unipotent radical of $B$. The set $X(T)$ of additive characters for $T$ can be seen as a subset of $\mathbb{R}^{m}$ with basis $\left\{\omega_{1}, \omega_{2}, \cdots, \omega_{l}\right\}$ satisfying $\left\langle\omega_{i}, \alpha_{j}\right\rangle=\delta_{i j}$. The set $X(T)$ also has the following property:

$$
X(T)=\left\{\lambda \in \mathbb{R}^{m} \mid\langle\lambda, \alpha\rangle \in \mathbb{Z} \text { for all } \alpha \in R\right\} .
$$

Let $X(T)^{+}=\left\{\lambda \in X(T) \mid\langle\lambda, \alpha\rangle \geq 0\right.$ for all $\left.\alpha \in R^{+}\right\}$be the set of dominant weights, and let $X_{1}(T)=\left\{\lambda \in X(T)^{+} \mid 0 \leq\langle\lambda, \alpha\rangle<p\right.$ for all $\left.\alpha \in \Delta\right\}$ be the set of restricted weights.

Let $\lambda \in X(T)^{+}$and $H^{0}(\lambda)=\left\{f \in k[G] \mid f(g b)=\lambda(b)^{-1} f(g)\right.$ for all $\left.g \in G, b \in B\right\}$, where $k[G]$ is the algebra of all regular functions on $G$. The action of $G$ on $H^{0}(\lambda)$ is defined by $g f(h)=f\left(g^{1} h\right)$, where $f \in H^{0}(\lambda), g, h \in G$ [35] (p. 26). On the other hand, $H^{0}(\lambda)=\operatorname{Ind}_{B}^{G}\left(k_{\lambda}\right)$, where $k_{\lambda}$ is a one-dimensional $B$-module defined by $\lambda \in X(T)^{+}$via the isomorphism $B / U \cong T$ [35] (p. 176). Let $L(\lambda)$ be a maximal semi-simple submodule (socle) of $H^{0}(\lambda)$. If $H^{0}(\lambda) \neq 0$, then $L(\lambda)$ is simple [35] (p. 177, II.2.3) and every simple G-module is isomorphic to $L(\lambda)$ for some $\lambda \in X(T)^{+}$[35] (p. 177, II.2.4). Since $H^{0}(\lambda) \neq 0$ for all $\lambda \in X(T)^{+}$[35] (p. 178, II.2.6), then, for all $\lambda \in X(T)^{+}$, there is a short exact sequence $0 \rightarrow L(\lambda) \rightarrow H^{0}(\lambda) \rightarrow H^{0}(\lambda) / L(\lambda) \rightarrow 0$ of $G$-modules. One of the effective ways to explicitly describe the structure of $H^{0}(\lambda) / L(\lambda)$ is to study the radical of the Weyl module $V(\lambda)$ with the highest weight $\lambda \in X(T)^{+}$. The Weyl module $V(\lambda)$ is isomorphic to $H^{0}\left(-w_{0}(\lambda)\right)^{*}$, where $w_{0}$ is the maximal element of the Weyl group $W$ for $R$ [35] (p. 182, II.2.13). So, for all $\lambda \in X(T)^{+}$, there is a short exact sequence $0 \rightarrow \operatorname{rad} V(\lambda) \rightarrow V(\lambda) \rightarrow L(\lambda) \rightarrow 0$ of $G$ modules, where rad $V(\lambda)$ is the radical of $V(\lambda)$. For the Lie algebra $\mathfrak{g}$ of $G$, we will consider 
the corresponding differentials of the $G$-modules $H^{0}(\lambda), V(\lambda)$ and $L(\lambda)$. We denote these $\mathfrak{g}$-modules also via $H^{0}(\lambda), V(\lambda)$ and $L(\lambda)$, respectively. In the restricted region, these three $\mathfrak{g}$-modules are restricted; moreover, $L(\lambda)$ remains simple as a $\mathfrak{g}$-module.

For $\alpha \in R^{+}$and $n \in \mathbb{Z}$ let us define the affine reflections $s_{\alpha, n}$ on $X(T)$ by

$$
s_{\alpha, n} \cdot \lambda=\lambda-\langle\lambda+\rho, \alpha\rangle \alpha+n p \alpha \text { for all } \lambda \in X(T) .
$$

Denote by $W_{p}$ the affine Weyl group generated by all $s_{\alpha, n}$ with $\alpha \in R^{+}$and $n \in \mathbb{Z}$. The finite Weyl group $W$ of $R$ appears as the subgroup of $W_{p}$ generated by the reflections $s_{\alpha, 0}$ with $\alpha \in R^{+}$.

Let $\alpha_{0}$ be the unique maximal short root of $R$. We will use the following short notation: $s_{\alpha_{i}, 0} s_{i}$ for all $i \in\{1,2, \cdots, l\}$ and $s_{0} s_{\alpha_{0}, 1}$. The set of simple reflections in $W$ is $S=\left\{s_{i} \mid i=1,2, \cdots, l\right\}$ and the set of simple affine reflections in $W_{p}$ is $S_{p}=S \cup\left\{s_{0}\right\}$. Denote by $l(w)$ the length of the element $w \in W$ with respect to the simple reflections $s_{1}, s_{2}, \cdots, s_{l}$.

We use an order relation $\uparrow$ on $X(T)$, as described in [35] (p. 235). Let $\lambda, \mu \in X(T)$. We say $\lambda \uparrow \mu$ will hold if and only if there are $v_{1}, v_{2}, \cdots, v_{t} \in X(T)$ and reflections $r_{1}, r_{2}, \cdots, r_{t+1} \in W_{p}$ with

$$
\lambda \leq r_{1} \cdot \lambda=v_{1} \leq r_{1} \cdot v_{1}=v_{2} \leq \cdots \leq r_{t} \cdot v_{t-1}=v_{t} \leq r_{t+1} \cdot v_{t}=\mu
$$

or if $\mu=\lambda$. If $\lambda \in X(T)$ and $\alpha \in R^{+}$. Then, there are unique $n_{\alpha}, d_{\alpha} \in \mathbb{Z}$ with

$$
\langle\lambda+\rho, \alpha\rangle=n_{\alpha} p+d_{\alpha}
$$

and $0<d_{\alpha} \leq p$. Now, set $d(\lambda)=\sum_{\alpha \in R^{+}} n_{\alpha}$.

For a vector space $L$ over $k$, we denote by $L^{(1)}$ the vector space over $k$ that coincides with $L$ as an additive group and with the scalar multiplication given by $a \cdot v=\sqrt[p]{a} v$ for all $a \in k, v \in L$, where the left hand side is the new multiplication and the right hand side the old one. If $L$ is a $G$-module, then $L^{(1)}$ is also a $G$-module using the given action of any $g \in G$ on the additive group $L^{(1)}=L$. The new $G$-module $L^{(1)}$ is called the Frobenius twist of $L$. Suppose a $G$-module $V$ is a Frobenius twist. Then, there is a unique rational $G$-module $L$ such that $L^{(1)}=V$. Denote this module by $V^{(-1)}$.

\subsection{Calculation Algorithm of the Cohomology with Coefficients in Simple Modules}

To calculate the cohomology $H^{n}(\mathfrak{g}, L(\lambda))$, we will use the following known facts:

- The connection between the cohomology $H^{n}\left(G_{1}, V\right)$ and $H^{n}(\mathfrak{g}, \mathrm{V})$, where $V$ is a restricted $\mathfrak{g}$-module (or $G_{1}$-module) introduced by Friedlander, Parshall and Farnsteiner $[18,19]$ as the spectral sequence

$$
\oplus_{i+j=n} \operatorname{Hom}_{k}\left(\wedge^{i}(\mathfrak{g}), H^{j}\left(G_{1}, V\right)\right) \Rightarrow H^{n}(\mathfrak{g}, \mathrm{V}) .
$$

We use the following special cases of the spectral sequence (1) (see, Theorem 3.1 in [36]): (1) If $V$ is a restricted $\mathfrak{g}$-module, then we have for every $n \geq 0$ :

$$
H^{j}(\mathfrak{g}, \mathrm{V})=0 \forall j \leq n \text { if and only if } H^{j}\left(G_{1}, \mathrm{~V}\right)=0 \forall j \leq n ;
$$

(2) If $H^{j}(\mathfrak{g}, \mathrm{V})=0 \forall j \leq n-1$, then

$$
H^{n}(\mathfrak{g}, \mathrm{V}) \cong H^{n}\left(G_{1}, \mathrm{~V}\right) .
$$

- Andersen-Janzen Formula on cohomology of $G_{1}$ with coefficients in $H^{0}(\lambda)$, see [37]: let $p>h$, and $\lambda=w \cdot 0+p v$, then:

$$
H^{i}\left(G_{1}, H^{0}(\lambda)\right)^{(-1)} \cong\left\{\begin{array}{cl}
\operatorname{Ind}_{B}^{G}\left(S^{(i-l(w)) / 2} \mathfrak{n}^{*} \otimes k_{v}\right) & \text { if } i-l(w) \text { even } \\
0 & \text { if } i-l(w) \text { odd }
\end{array}\right.
$$


where $\mathfrak{n}$ is the maximal nilpotent subalgebra of $\mathfrak{g}$, corresponding to the negative roots. The Lie algebra $\mathfrak{n}$ is the Lie algebra of the unipotent radical $U$ of $B$.

For the cohomology $H^{n}(\mathfrak{g}, L(\lambda))$ we use the following calculation algorithm:

(A1) Calculate $H^{n}\left(G_{1}, H^{0}(\lambda)\right)^{(-1)}$ using the Formula (4);

(A2) Transition from $H^{n}\left(G_{1}, H^{0}(\lambda)\right)^{(-1)}$ to $H^{n}\left(\mathfrak{g}, H^{0}(\lambda)\right)$ using the special cases (2) and (3) of the spectral sequence (1) for $V=H^{0}(\lambda)$;

(A3) Transition from $H^{n}\left(\mathfrak{g}, H^{0}(\lambda)\right)$ to $H^{n}(\mathfrak{g}, L(\lambda))$ using the long cohomological sequence

$$
\rightarrow H^{n}(\mathfrak{g}, L(\lambda)) \rightarrow H^{n}\left(\mathfrak{g}, H^{0}(\lambda)\right) \rightarrow H^{n}\left(\mathfrak{g}, H^{0}(\lambda) / L(\lambda)\right) \rightarrow
$$

corresponding to the short exact sequence $0 \rightarrow L(\lambda) \rightarrow H^{0}(\lambda) \rightarrow H^{0}(\lambda) / L(\lambda) \rightarrow 0$.

Consider an example of the application of the algorithm (A1)-(A3). Let $\mathfrak{g}$ be the classical Lie algebra of type $B_{3}$ over an algebraically closed field $k$ of characteristic $p>5$ and $\lambda=(p-5) \omega_{1}$.

(B1) First, we will calculate $H^{n}\left(G_{1}, H^{0}\left((p-5) \omega_{1}\right)^{(-1)}\right.$ using the Formula (4). Since $(p-5) \omega_{1}=s_{1} s_{2} s_{3} s_{2} s_{1} \cdot 0+p \omega_{1}$ and $l\left(s_{1} s_{2} s_{3} s_{2} s_{1}\right)=5$, then by $(4)$,

$$
H^{n}\left(G_{1}, H^{0}\left((p-5) \omega_{1}\right)^{(-1)}=\left\{\begin{array}{c}
0 \quad \text { if } n<4, \\
L\left(\omega_{1}\right) \text { if } n=5 .
\end{array}\right.\right.
$$

(B2) Then, by (3),

$$
H^{n}\left(\mathfrak{g}, H^{0}\left((p-5) \omega_{1}\right)^{(-1)}=\left\{\begin{array}{c}
0 \quad \text { if } n<4 \\
L\left(\omega_{1}\right) \text { if } n=5 .
\end{array}\right.\right.
$$

(B3) There is short exact sequence $0 \rightarrow L\left((p-5) \omega_{1}\right) \rightarrow H^{0}\left((p-5) \omega_{1}\right) \rightarrow k \rightarrow 0$. Consider the corresponding long exact cohomological sequence

$$
\begin{aligned}
0 & \rightarrow H^{0}\left(\mathfrak{g}, L\left((p-5) \omega_{1}\right)\right) \rightarrow H^{0}\left(\mathfrak{g}, H^{0}\left((p-5) \omega_{1}\right)\right) \rightarrow H^{0}(\mathfrak{g}, k) \\
& \rightarrow H^{1}\left(\mathfrak{g}, L\left((p-5) \omega_{1}\right)\right) \rightarrow H^{1}\left(\mathfrak{g}, H^{0}\left((p-5) \omega_{1}\right)\right) \rightarrow H^{1}(\mathfrak{g}, k)
\end{aligned}
$$

It is obvious that $H^{0}\left(\mathfrak{g}, L\left((p-5) \omega_{1}\right)\right)=0$ and $H^{0}(\mathfrak{g}, k) \cong k$. Then, according to (B2), the last exact sequence gives the following split short exact sequences:

$$
\begin{aligned}
& \rightarrow k \rightarrow H^{1}\left(\mathfrak{g}, L\left((p-5) \omega_{1}\right)\right) \rightarrow 0 \\
& 0 \rightarrow H^{1}(\mathfrak{g}) \rightarrow H^{2}\left(\mathfrak{g}, L\left((p-5) \omega_{1}\right)\right) \rightarrow 0 \\
& 0 \rightarrow H^{2}(\mathfrak{g}) \rightarrow H^{3}\left(\mathfrak{g}, L\left((p-5) \omega_{1}\right)\right) \rightarrow 0 \\
& 0 \rightarrow H^{3}(\mathfrak{g}) \rightarrow H^{4}\left(\mathfrak{g}, L\left((p-5) \omega_{1}\right)\right) \rightarrow 0 \\
& 0 \rightarrow H^{4}(\mathfrak{g}) \rightarrow H^{5}\left(\mathfrak{g}, L\left((p-5) \omega_{1}\right)\right) \rightarrow H^{5}\left(\mathfrak{g}, H^{0}\left((p-5) \omega_{1}\right)\right) \rightarrow 0,
\end{aligned}
$$

According to Appendix A (Example A2), $H^{n}(\mathfrak{g})=0$ if $n=0,1,2,4$, and $H^{3}(\mathfrak{g})=k$. Therefore,

$$
H^{n}\left(\mathfrak{g}, L\left((p-5) \omega_{1}\right)^{(-1)}=\left\{\begin{array}{c}
0 \text { if } n=0,2,3 \\
k \text { if } n=1,4 \\
L\left(\omega_{1}\right) \text { if } n=5 .
\end{array}\right.\right.
$$


Similar calculations for $(p-5) \omega_{1}+\omega_{2}=s_{1} s_{2} s_{3} s_{2} \cdot 0+p \omega_{1}$ give us the following

$$
H^{n}\left(\mathfrak{g}, L\left((p-5) \omega_{1}+\omega_{2}\right)^{(-1)}=\left\{\begin{array}{c}
0 \text { if } n=0,1,3 \\
k \text { if } n=2, \\
L\left(\omega_{1}\right) \text { if } n=4 .
\end{array}\right.\right.
$$

\section{Results}

\subsection{Formulation of Results}

Consider the dominant weights $\lambda_{0}, \lambda_{1}, \cdots, \lambda_{s}, \mu_{1}, \mu_{2}, \cdots, \mu_{s-1}$, where

$$
s=\left\{\begin{array}{c}
l-1 \text { if } R=A_{l}, D_{l}, E_{l}(l=6,7,8), \\
2 l-2 \text { if } R=B_{l}, C_{l}, \\
3 \text { if } R=F_{4} \\
2 \text { if } R=G_{2}
\end{array}\right.
$$

introduced in $[29,30]$. Let us give a short description of them. Let $\left\{\beta_{i}\right\}_{i=1}^{s}$ be a sequence of positive roots giving rise to $s_{\beta_{1}, k(1)}, s_{\beta_{2}, k(2)}, \cdots, s_{\beta_{s}, k(s)}$, sequence of reflections in $W_{p}$. Then $\left\{\lambda_{i}\right\}_{i=1}^{s}$ is a sequence of dominant weights, which satisfy:

1. $\lambda_{0}=0$, and for $1 \leq i \leq s, \lambda_{i}=s_{\beta_{i}, k(i)} \cdot \lambda_{i-1}$;

2. $\quad \lambda_{0} \uparrow \lambda_{1} \uparrow \cdots \uparrow \lambda_{s}$ and $d\left(\lambda_{i}\right)=i, 1 \leq i \leq s ;$

3. $\left\langle\lambda_{i}+\rho, \alpha\right\rangle<p^{2}$ for all $i$ and for all $\alpha \in R^{+}$;

4. for each pair $i, j$ with $i>j$ there exists a positive root $\gamma(i, j)$ such that, for all $i$, $\sum_{j=1}^{i-1} s_{\beta_{j}, k(j)} s_{\beta_{i}, k(i)}=\sum_{j=1}^{i-1} s_{\gamma(i, j)} s_{\beta_{j}, k(j)}$ as element of $\mathbb{Z}\left[W_{p}\right]$.

Further, let $\gamma_{0}$ be a positive root. Then $\left\{\mu_{i}\right\}_{i=1}^{s-1}$ is a sequence of dominant weights, which satisfy:

5. $\mu_{i}=s_{\gamma_{0}, 1} \cdot \lambda_{i+1}, 1 \leq i \leq s-1$;

6. $\quad \lambda_{i+1} \uparrow \mu_{i}, 1 \leq i \leq s-1$;

7. $\quad \mu_{1} \uparrow \mu_{2} \uparrow \cdots \uparrow \mu_{s-1}$ and $d\left(\mu_{i}\right)=i+2,1 \leq i \leq s-1$;

8. $\left\langle\mu_{i}+\rho, \alpha\right\rangle<p^{2}$ for all $i$ and for all $\alpha \in R^{+}$;

9. for $j \in\{1,2\}$ there exists a positive root $\gamma(j)$ such that

$$
\sum_{j=1}^{2} s_{\beta_{j}, k(j)} s_{\gamma_{0}, 1}=\sum_{j=1}^{2} s_{\gamma(j)} s_{\beta_{j}, k(j)} ;
$$

10. $\left\langle\gamma_{0}, \beta_{i}\right\rangle=0$, for $3 \leq i \leq s-1$.

For the cohomology of simple modules with highest weights $\lambda_{0}, \lambda_{1}, \cdots, \lambda_{s}$, $\mu_{1}, \mu_{2}, \cdots, \mu_{s-1}$, the following results hold:

Theorem 1. Let $\mathfrak{g}$ be a Lie algebra of classical type over an algebraically closed field $k$ of characteristic $p>h$, where $h$ is the Coxeter number. Suppose that $\left\{\lambda_{i}\right\}_{i=1}^{s}$ a sequence of dominant weights, which satisfy the conditions (1)-(4), and $\lambda_{i}=w_{i} \cdot 0+p v_{i}$, where $w_{i} \in W, v_{i} \in X(T)^{+}$, and $i \in\{1,2, \cdots, s\}$. Then

(a) for all $i \in\left\{1,2, \cdots, t_{\lambda}\right\}$,

$$
H^{n}\left(\mathfrak{g}, L\left(\lambda_{i}\right)\right) \cong\left\{\begin{aligned}
0 & \text { if } n<i, \\
k & \text { if } n=i, \\
H^{n-i}(\mathfrak{g}) & \text { if } i<n<l\left(w_{i}\right),
\end{aligned}\right.
$$

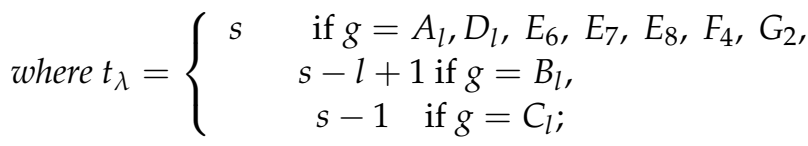


(b) if $\mathfrak{g}=B_{l}$, then

$$
H^{n}\left(\mathfrak{g}, L\left(\lambda_{t_{\lambda}+1}\right)\right) \cong\left\{\begin{aligned}
& 0 \text { if } n<t_{\lambda}+1 \\
& L\left(\alpha_{0}\right)^{(1)} \oplus k \text { if } n<t_{\lambda}+1
\end{aligned}\right.
$$

and for all $i \in\left\{t_{\lambda}+2, t_{\lambda}+2, \cdots, s\right\}$,

$$
H^{n}\left(\mathfrak{g}, L\left(\lambda_{i}\right)\right) \cong\left\{\begin{array}{c}
0 \text { if } n<2 l-i \\
L\left(\alpha_{0}\right)^{(1)} \text { if } n=2 l-i
\end{array}\right.
$$

(c) if $\mathfrak{g}=C_{l}$, then

$$
H^{n}\left(\mathfrak{g}, L\left(\lambda_{s}\right)\right) \cong\left\{\begin{aligned}
& 0 \text { if } n<s \\
& L\left(\alpha_{0}\right)^{(1)} \oplus k \text { if } n=s
\end{aligned}\right.
$$

Theorem 2. Let $\mathfrak{g}$ be a Lie algebra of classical type over an algebraically closed field $k$ of characteristic $p>h$, where $h$ is the Coxeter number. Suppose that $\left\{\mu_{j}\right\}_{j=1}^{s-1}$ a sequence of dominant weights, which satisfy the conditions (5)-(10), and $\mu_{j}=u_{j} \cdot 0+p \delta_{j}$, where $u_{j} \in W, \delta_{j} \in X(T)^{+}$, and $j \in\{1,2, \cdots, s-1\}$. Then

(a) $H^{n}\left(\mathfrak{g}, L\left(\mu_{1}\right)\right) \cong\left\{\begin{array}{c}0 \text { if } n<3, \\ \text { k } \text { if } n=3, \\ H^{n-3}(\mathfrak{g}) \text { if } 3<n<l\left(w_{2}\right)+1 ;\end{array}\right.$

(b) for all $i \in\left\{2,3, \cdots, t_{\mu}\right\}$,

$$
H^{n}\left(\mathfrak{g}, L\left(\mu_{j}\right)\right) \cong\left\{\begin{array}{c}
0 \text { if } n<j \text { and } n=j+1, \\
k \text { if } n=j, j+2 \\
H^{n-j}(\mathfrak{g}) \oplus H^{n-j-2}(\mathfrak{g}) \text { if } j+2<n<l\left(u_{j}\right),
\end{array}\right.
$$

where $t_{\mu}=\left\{\begin{array}{c}s-1 \text { if } \mathfrak{g}=A_{l}, B_{l}, D_{l}, \\ s-2 \text { if } \mathfrak{g}=C_{l}\end{array}\right.$

(c) if $\mathfrak{g}=C_{l}$, then

$$
H^{n}\left(\mathfrak{g}, L\left(\mu_{s-1}\right)\right) \cong\left\{\begin{array}{cc}
0 & \text { if } n<s-1 \\
L\left(\alpha_{0}\right) & (1) \oplus k \text { if } n=s-1 .
\end{array}\right.
$$

Remark 1. In the case of exceptional Lie algebras $\left(\mathfrak{g}=E_{6}, E_{7}, E_{8}, F_{4}, G_{2}\right)$, dominant weights $\mu_{1}, \mu_{2}, \cdots, \mu_{s-1}$, satisfying conditions (5)-(10) do not exist.

Corollary 3. Let $\mathfrak{g}$ be a Lie algebra of classical type over an algebraically closed field $k$ of characteristic $p>h$, where $h$ is the Coxeter number. Let $G$ be a semisimple, simply connected algebraic group with the Lie algebra $\mathfrak{g}$ and $G_{1}$ be the first Frobenius kernel of $G$. Suppose that $\left\{\lambda_{i}\right\}_{i=1}^{s}$ a sequence of dominant weights, which satisfy the conditions (1)-(4), and $i \in\left\{1,2, \cdots, t_{\lambda}\right\}$. Then the following non-trivial isomorphisms hold: $H^{i}\left(\mathfrak{g}, L\left(\lambda_{i}\right)\right) \cong H^{i}\left(G_{1}, L\left(\lambda_{i}\right)\right) \cong H^{i}\left(G, L\left(\lambda_{i}\right)\right)$.

\subsection{Proof of the Results}

Proof of Theorem 1. We will first prove that, for all $i \in\{0,1, \cdots, s\}$, the dominant weight $\lambda_{i}$ can be represented in the form $\lambda_{i}=w_{i} \cdot 0+p v_{i}$, with $w_{i} \in W$ and $v_{i} \in X(T)^{+}$. For each of the root systems $R$ the description of the dominant weights $\lambda_{0}, \lambda_{1}, \cdots, \lambda_{s}$ by the generators $s_{0}, s_{1}, \cdots, s_{l}$ of the affine Weyl group $W_{p}$ is given in Table 1. 
Table 1. Description of the highest weights by the generators of $W_{p}$.

\begin{tabular}{|c|c|}
\hline $\begin{array}{l}\text { Root } \\
\text { System }\end{array}$ & Highest Weights \\
\hline$A_{l}$ & $\begin{array}{c}\lambda_{0}=1 \cdot \lambda_{0}, \lambda_{1}=s_{0} \cdot \lambda_{0}, \cdots, \lambda_{l-1}=s_{0} s_{1} \cdots s_{l-2} \cdot \lambda_{0} \\
\mu_{1}=s_{0} s_{l} \cdot \lambda_{0}, \mu_{2}=s_{0} s_{1} s_{l} \cdot \lambda_{0}, \cdots, \mu_{l-2}=s_{0} s_{1} \cdots s_{l-3} s_{l} \cdot \lambda_{0}\end{array}$ \\
\hline$B_{l}$ & $\begin{array}{c}\lambda_{0}=1 \cdot \lambda_{0}, \lambda_{1}=s_{0} \cdot \lambda_{0}, \cdots, \lambda_{l+1}=s_{0} s_{1} \cdots s_{l} \cdot \lambda_{0} \\
\lambda_{l+2}=s_{0} s_{1} \cdots s_{l} s_{l-1} \cdot \lambda_{0}, \cdots, \lambda_{2 l-2}=s_{0} s_{1} \cdots s_{l} s_{l-1} s_{l-2} \cdots s_{3} \cdot \lambda_{0} \\
\mu_{1}=s_{0} s_{1} s_{0} \cdot \lambda_{0}, \mu_{2}=s_{0} s_{1} s_{2} s_{0} \cdot \lambda_{0}, \cdots, \mu_{l}=s_{0} s_{1} \cdots s_{l} s_{0} \cdot \lambda_{0} \\
\mu_{l+1}=s_{0} s_{1} \cdots s_{l} s_{l-1} s_{0} \cdot \lambda_{0}, \cdots, \mu_{2 l-3}=s_{0} s_{1} \cdots s_{l} s_{l-1} s_{l-2} \cdots s_{3} s_{0} \cdot \lambda_{0} .\end{array}$ \\
\hline$C_{l}$ & $\begin{array}{c}\lambda_{0}=1 \cdot \lambda_{0}, \lambda_{1}=s_{0} \cdot \lambda_{0}, \lambda_{2}=s_{0} s_{2} \cdot \lambda_{0}, \cdots, \lambda_{l}=s_{0} s_{2} \cdots s_{l} \cdot \lambda_{0} \\
\lambda_{l+1}=s_{0} s_{2} \cdots s_{l} s_{l-1} \cdot \lambda_{0}, \cdots, \lambda_{2 l-2}=s_{0} s_{2} \cdots s_{l} s_{l-1} s_{l-2} \cdots s_{2} \cdot \lambda_{0} \\
\mu_{1}=s_{0} s_{2} s_{1} \cdot \lambda_{0}, \mu_{2}=s_{0} s_{2} s_{3} s_{1} \cdot \lambda_{0}, \cdots, \mu_{l-1}=s_{0} s_{2} \cdots s_{l} s_{1} \cdot \lambda_{0} \\
\mu_{l}=s_{0} s_{2} \cdots s_{l} s_{l-1} s_{1} \cdot \lambda_{0}, \cdots, \mu_{2 l-3}=s_{0} s_{2} \cdots s_{l} s_{l-1} s_{l-2} \cdots s_{2} s_{1} \cdot \lambda_{0}\end{array}$ \\
\hline$D_{l}$ & $\begin{array}{c}\lambda_{0}=1 \cdot \lambda_{0}, \lambda_{1}=s_{0} \cdot \lambda_{0}, \lambda_{2}=s_{0} s_{2} \cdot \lambda_{0}, \cdots, \lambda_{l-1}=s_{0} s_{2} \cdots s_{l-1} \cdot \lambda_{0} \\
\mu_{1}=s_{0} s_{2} s_{1} \cdot \lambda_{0}, \mu_{2}=s_{0} s_{2} s_{3} s_{1} \cdot \lambda_{0}, \cdots, \mu_{l-2}=s_{0} s_{2} \cdots s_{l-1} s_{1} \cdot \lambda_{0}\end{array}$ \\
\hline$E_{6}$ & $\begin{array}{c}\lambda_{0}=1 \cdot \lambda_{0}, \lambda_{1}=s_{0} \cdot \lambda_{0}, \lambda_{2}=s_{0} s_{2} \cdot \lambda_{0}, \lambda_{3}=s_{0} s_{2} s_{4} \cdot \lambda_{0} \\
\lambda_{4}=s_{0} s_{2} s_{4} s_{5} \cdot \lambda_{0}, \lambda_{5}=s_{0} s_{2} s_{4} s_{5} s_{6} \cdot \lambda_{0}\end{array}$ \\
\hline$E_{7}$ & $\begin{array}{c}\lambda_{0}=1 \cdot \lambda_{0}, \lambda_{1}=s_{0} \cdot \lambda_{0}, \lambda_{2}=s_{0} s_{1} \cdot \lambda_{0}, \lambda_{3}=s_{0} s_{1} s_{3} \cdot \lambda_{0} \\
\lambda_{4}=s_{0} s_{1} s_{3} s_{4} \cdot \lambda_{0}, \lambda_{5}=s_{0} s_{1} s_{3} s_{4} s_{5} \cdot \lambda_{0}, \lambda_{6}=s_{0} s_{1} s_{3} s_{4} s_{5} s_{6} \cdot \lambda_{0}\end{array}$ \\
\hline$E_{8}$ & $\begin{array}{c}\lambda_{0}=1 \cdot \lambda_{0}, \lambda_{1}=s_{0} \cdot \lambda_{0}, \lambda_{2}=s_{0} s_{8} \cdot \lambda_{0}, \lambda_{3}=s_{0} s_{8} s_{7} \cdot \lambda_{0}, \\
\lambda_{4}=s_{0} s_{8} s_{7} s_{6} \cdot \lambda_{0}, \lambda_{5}=s_{0} s_{8} s_{7} s_{6} s_{5} \cdot \lambda_{0}, \lambda_{6}=s_{0} s_{8} s_{7} s_{6} s_{5} s_{4} \cdot \lambda_{0}, \lambda_{7}=s_{0} s_{8} s_{7} s_{6} s_{5} s_{4} s_{3} \cdot \lambda_{0},\end{array}$ \\
\hline$F_{4}$ & $\lambda_{0}=1 \cdot \lambda_{0}, \lambda_{1}=s_{0} \cdot \lambda_{0}, \lambda_{2}=s_{0} s_{4} \cdot \lambda_{0}, \lambda_{3}=s_{0} s_{4} s_{3} \cdot \lambda_{0}$ \\
\hline$G_{2}$ & $\lambda_{0}=1 \cdot \lambda_{0}, \lambda_{1}=s_{0} \cdot \lambda_{0}, \lambda_{2}=s_{0} s_{1} \cdot \lambda_{0}$ \\
\hline
\end{tabular}

We have $\lambda_{1}=s_{0} \cdot 0=w_{1} \cdot 0+p v_{1}$. Since

$$
s_{0} \cdot 0=(p-h+1) \alpha_{0},
$$

where $\alpha_{0}$ is the maximal short root, the equality $w_{1} \cdot 0+p v_{1}=(p-h+1) \alpha_{0}$ gives us

$$
w_{1}^{-1} \cdot\left((-h+1) \alpha_{0}\right)=0 \text {. }
$$

Using Equations (6) and (7), one can easy to get the descriptions of $w_{1}$ and $s_{0}$ by the generators $s_{0}, s_{1}, \cdots, s_{l}$ of the Weyl group $W$. For non-negative integer indices $i<j$, let

$$
w_{i, j}=s_{i} s_{i+1} \cdots s_{j} .
$$

Simple calculations for each of the root systems yield $\nu_{1}=\alpha_{0}$ and $s_{0}=w_{1, l} w_{1, l-1}^{-1}+p \alpha_{0}$ for $\mathfrak{g}=A_{l}, B_{l}$;

$s_{0}=w_{2, l-1} w_{1, l}^{-1} w_{2, l-1} w_{2, l}^{-1}+p \alpha_{0}$ for $\mathfrak{g}=C_{l}$;

$s_{0}=w_{2, l-2} w_{1, l}^{-1} w_{2, l-2} w_{2, l}^{-1}+p \alpha_{0}$ for $\mathfrak{g}=D_{l}$;

$s_{0}=s_{2} w_{4,6}^{-1} w_{3,5} w_{1,2}^{-1} w_{3,4}^{-1} s_{1} w_{4,6} s_{2} w_{4,5} w_{3,4} s_{2}+p \alpha_{0}$ for $\mathfrak{g}=E_{6}$;

$s_{0}=s_{1} w_{2,4}^{-1} w_{4,5}^{-1} w_{5,6}^{-1} w_{3,4} w_{1,3} w_{1,7}^{-1} w_{3,5}^{-1} w_{4,6}^{-1} s_{2} w_{3,7}^{-1} s_{1}+p \alpha_{0}$ for $\mathfrak{g}=E_{7} ;$

$s_{0}=w_{1,8}^{-1} w_{3,4}^{-1} w_{4,5}^{-1} s_{2} w_{3,6}^{-1} s_{1} w_{2,7}^{-1} w_{1,8}^{-1} w_{3,5}^{-1} w_{4,6}^{-1} s_{2} w_{3,7}^{-1} w_{4,8}^{-1} w_{1,8}+p \alpha_{0}$ for $\mathfrak{g}=E_{8}$;

$s_{0}=w_{1,4}^{-1} w_{2,3}^{-1} w_{1,4}^{-1} w_{2,4}^{-1} w_{3,4}+p \alpha_{0}$ for $\mathfrak{g}=F_{4}$;

$s_{0}=w_{1,2} w_{1,2} s_{1}+p \alpha_{0}$ for $\mathfrak{g}=G_{2}$.

Then using Table 1 and above expression of $s_{0}$, for $w_{i}$ and $v_{i}$, we obtain the expressions that shown in Table 2. 
Table 2. Descriptions of $w_{i}$ and $v_{i}$.

\begin{tabular}{|c|c|c|c|}
\hline $\begin{array}{l}\text { Root } \\
\text { System }\end{array}$ & $i$ & $w_{i}$ & $v_{i}$ \\
\hline$A_{l}$ & $1,2, \cdots, s$ & $w_{1, l} w_{i, l-1}^{-1}$ & $\alpha_{0}$ \\
\hline$B_{l}$ & $\begin{array}{c}1,2, \cdots, l \\
l+1, l+2, \cdots, s\end{array}$ & $\begin{array}{c}w_{1, l} w_{i, l-1}^{-1} \\
w_{1,2 l-i}\end{array}$ & $\alpha_{0}$ \\
\hline$C_{l}$ & $\begin{array}{l}1,2, \cdots, l-1 \\
l, l+1, \cdots, s\end{array}$ & $\begin{array}{c}w_{2, l} w_{1, l-1}^{-1} w_{2, l} w_{i+1, l-1}^{-1} \\
w_{2, l} w_{1, l-1}^{-1} w_{2,2 l-i-1} w_{1, l-1}^{-1}\end{array}$ & $\alpha_{0}$ \\
\hline$D_{l}$ & $1,2, \cdots, s$ & $w_{2, l-2} w_{1, l}^{-1} w_{2, l-2} w_{i+1, l}^{-1}$ & $\alpha_{0}$ \\
\hline$E_{6}$ & $\begin{array}{l}1 \\
2 \\
3 \\
4 \\
5\end{array}$ & $\begin{array}{c}s_{2} w_{4,6}^{-1} w_{3,5} w_{1,2}^{-1} w_{3,4}^{-1} s_{1} w_{4,6} s_{2} w_{4,5} w_{3,4} s_{2} \\
s_{2} w_{4,6}^{-1} w_{3,5} w_{1,2}^{-1} w_{3,4}^{-1} s_{1} w_{4,6} s_{2} w_{4,5} w_{3,4} \\
s_{2} w_{4,6}^{-1} w_{3,5} w_{1,2}^{-1} w_{3,4}^{-1} s_{1} w_{4,6} s_{2} w_{4,5} s_{3} \\
s_{2} w_{4,6}^{-1} w_{3,5} w_{1,2}^{-1} w_{3,4}^{-1} s_{1} w_{4,6} s_{2} w_{3,4}^{-1} \\
s_{2} w_{4,6}^{-1} w_{3,5} w_{1,2}^{-1} w_{3,4}^{-1} s_{1} w_{4,5} s_{2} w_{3,4}^{-1}\end{array}$ & $\alpha_{0}$ \\
\hline$E_{7}$ & $\begin{array}{c}1 \\
2,3,4,5 \\
6\end{array}$ & $\begin{array}{c}s_{1} w_{2,4}^{-1} w_{4,-1}^{-1} w_{5,6}^{-1} w_{3,4} w_{1,3} w_{1,7}^{-1} w_{3,5}^{-1} w_{4,-}^{-1} s_{2} w_{3,7}^{-1} s_{1} \\
s_{1} w_{2,4}^{-1} w_{4,5}^{-1} w_{5,6}^{-1} w_{3,4} w_{1,3} w_{1,7}^{-1} w_{3,5}^{-1} w_{4,6}^{-1} s_{2} w_{i+1,7}^{-1} \\
s_{1} w_{2,4}^{-1} w_{4,5}^{-1} w_{5,6}^{-1} w_{3,4} w_{1,3} w_{1,7}^{-1} w_{3,5}^{-1} w_{4,6}^{-1} s_{2} s_{7}\end{array}$ & $\alpha_{0}$ \\
\hline$E_{8}$ & $1,2, \cdots, 7$ & $w_{1,8}^{-1} w_{3,4}^{-1} w_{4,5}^{-1} s_{2} w_{3,6}^{-1} s_{1} w_{2,7}^{-1} w_{1,8}^{-1} w_{3,5}^{-1} w_{4,6}^{-1} s_{2} w_{3,7}^{-1} w_{4,8}^{-1} w_{1,9-i}$ & $\alpha_{0}$ \\
\hline$F_{4}$ & $\begin{array}{l}1 \\
2 \\
3\end{array}$ & $\begin{array}{c}w_{1,4}^{-1} w_{2,3}^{-1} w_{1,4}^{-1} w_{2,4}^{-1} w_{3,4} \\
w_{1,4}^{-1} w_{2,3}^{-1} w_{1,4}^{-1} w_{2,4}^{-1} s_{3} \\
w_{1,4}^{-1} w_{2,3}^{-1} w_{1,4}^{-1} w_{2,4}^{-1}\end{array}$ & $\alpha_{0}$ \\
\hline$G_{2}$ & $\begin{array}{l}1 \\
2\end{array}$ & $\begin{array}{c}w_{1,2} w_{1,2} s_{1} \\
w_{1,2} w_{1,2}\end{array}$ & $\alpha_{0}$ \\
\hline
\end{tabular}

Since $l\left(w_{i, j}\right)=j-i+1$, using Table 2 , for all $i \in\{1,2, \cdots, s\}$, we get

$l\left(w_{i}\right)=2 l-i$ for $\mathfrak{g}=A_{l}, B_{l}$;

$l\left(w_{i}\right)=4 l-i-4$ for $\mathfrak{g}=C_{l}$;

$l\left(w_{i}\right)=4 l-i-6$ for $\mathfrak{g}=D_{l}$;

$l\left(w_{i}\right)=22-i$ for $\mathfrak{g}=E_{6}$;

$l\left(w_{i}\right)=34-i$ for $\mathfrak{g}=E_{7}$;

$l\left(w_{i}\right)=58-i$ for $\mathfrak{g}=E_{8}$;

$l\left(w_{i}\right)=16-i$ for $\mathfrak{g}=F_{4}$;

$l\left(w_{i}\right)=6-i$ for $\mathfrak{g}=G_{2}$.

Now, we can calculate the cohomology $H^{n}\left(\mathfrak{g}, L\left(\lambda_{i}\right)\right)$ for all $i \in\{1,2, \cdots, s\}$ and for all $n<l\left(w_{i}\right)$ by the algorithm (A1)-(A3) described in Section 2.2.

Proof of Theorem 1 Statement (a). We have

(C1) According to Formula (4), $H^{n}\left(G_{1}, H^{0}\left(\lambda_{i}\right)\right)=0$ for all $i$ satisfying the condition $n<l\left(w_{i}\right)$.

(C2) By the above (C1) and Formula (2), $H^{n}\left(\mathfrak{g}, H^{0}\left(\lambda_{i}\right)\right)=0$ for all $i$ satisfying the condition $n<l\left(w_{i}\right)$.

(C3) Consider the long cohomological exact sequence (5) for $\lambda=\lambda_{i}$ :

$$
H^{n}\left(\mathfrak{g}, L\left(\lambda_{i}\right)\right) \rightarrow H^{n}\left(\mathfrak{g}, H^{0}\left(\lambda_{i}\right)\right) \rightarrow H^{n}\left(\mathfrak{g}, H^{0}\left(\lambda_{i}\right) / L\left(\lambda_{i}\right)\right)
$$

Since $H^{n}\left(\mathfrak{g}, H^{0}\left(\lambda_{i}\right)\right)=0$ for all $i$ satisfying the condition $n<l\left(w_{i}\right)$, from the exactness of the sequence $(8)$ it follows that

$$
H^{n}\left(\mathfrak{g}, L\left(\lambda_{i}\right)\right) \cong H^{n-1}\left(\mathfrak{g}, H^{0}\left(\lambda_{i}\right) / L\left(\lambda_{i}\right)\right)
$$

for all $i$ satisfying the condition $n<l\left(w_{i}\right)$. 
By Theorem 1.1 in [30] (pp. 3861—3862),

$$
H^{0}\left(\lambda_{i}\right) / L\left(\lambda_{i}\right) \cong L\left(\lambda_{i-1}\right)
$$

for all $i \in\{1,2, \cdots, s\}$. Then

$$
H^{n}\left(\mathfrak{g}, L\left(\lambda_{i}\right)\right) \cong H^{n-1}\left(\mathfrak{g}, L\left(\lambda_{i-1}\right)\right)
$$

for all $i$ satisfying the condition $n<l\left(w_{i}\right)$. If $i=t_{\lambda}+1$, then, by Formula (4),

$$
H^{t_{\lambda}+1}\left(G_{1}, H^{0}\left(\lambda_{t_{\lambda}+1}\right)\right) \neq 0 .
$$

Hence, it follows that $i \leq t_{\lambda}$.

Further, we will use induction on $i$. If $i=1$, then, by (9),

$$
H^{n}\left(\mathfrak{g}, L\left(\lambda_{1}\right)\right) \cong H^{n-1}\left(\mathfrak{g}, L\left(\lambda_{0}\right)\right)=H^{n-1}(\mathfrak{g})
$$

for all $n<l\left(w_{1}\right)=2 l-1$. Then

$$
H^{n}\left(\mathfrak{g}, L\left(\lambda_{1}\right)\right) \cong\left\{\begin{aligned}
0 & \text { if } n<1, \\
k & \text { if } n=1, \\
H^{n-1}(\mathfrak{g}) & \text { if } 1<n<l\left(w_{1}\right) .
\end{aligned}\right.
$$

Therefore, the statement $(a)$ is valid for $i=1$.

Suppose the statement (a) is valid for all $i<t$, where $t \leq t_{\lambda}$. By (9),

$$
H^{n}\left(\mathfrak{g}, L\left(\lambda_{t}\right)\right) \cong H^{n-1}\left(\mathfrak{g}, L\left(\lambda_{t-1}\right)\right)
$$

for all $n<l\left(w_{t}\right)=2 l-t$. Then, by the induction hypothesis,

$$
H^{n}\left(\mathfrak{g}, L\left(\lambda_{t}\right)\right) \cong\left\{\begin{aligned}
0 & \text { if } n<t, \\
k & \text { if } n=t, \\
H^{n-t}(\mathfrak{g}) & \text { if } t<n<l\left(w_{t}\right) .
\end{aligned}\right.
$$

Therefore, the statement $(a)$ is valid for all $i \in\left\{1,2, \cdots, t_{\lambda}\right\}$.

In the statement $(a)$, the following two cases are not covered: $\lambda_{i}$ with

$$
i \in\left\{t_{\lambda}+1, t_{\lambda}+2, \cdots, s\right\}
$$

for the Lie algebras of type $B_{l}$, and $\lambda_{s}$ for the Lie algebras of type $C_{l}$. Below we will consider them in the statements $(b)$ and $(c)$, respectively.

Proof of Theorem 1 Statement (b). Let $i=t_{\lambda}+1$. mula (4),

(D1) We get $\lambda_{t_{\lambda}+1}=w_{t_{\lambda}+1} \cdot 0+p \alpha_{0}$ and $l\left(w_{t_{\lambda}+1}\right)=t_{\lambda}+1$. Then, according to For-

$$
H^{n}\left(G_{1}, H^{0}\left(\lambda_{t_{\lambda}+1}\right)\right) \cong\left\{\begin{array}{cc}
0 & \text { if } n<t_{\lambda}+1 \\
L\left(\alpha_{0}\right)^{(1)} \text { if } n=t_{\lambda}+1
\end{array}\right.
$$

(D2) By the above (D1) and Formula (3),

$$
H^{n}\left(\mathfrak{g}, H^{0}\left(\lambda_{t_{\lambda}+1}\right)\right) \cong\left\{\begin{array} { c c } 
{ 0 } & { \text { if } n < t _ { \lambda } + 1 , } \\
{ H ^ { n } ( G _ { 1 } , H ^ { 0 } ( \lambda _ { t _ { \lambda } + 1 } ) ) \text { if } n = t _ { \lambda } + 1 }
\end{array} \cong \left\{\begin{array}{cc}
0 & \text { if } n<t_{\lambda}+1 \\
L\left(\alpha_{0}\right)^{(1)} & \text { if } n=t_{\lambda}+1 .
\end{array}\right.\right.
$$


(D3) Consider the long cohomological exact sequence (8) for $i=t_{\lambda}+1$. Since $H^{n}\left(\mathfrak{g}, H^{0}\left(\lambda_{t_{\lambda}+1}\right)\right)=0$ for all $n<t_{\lambda}+1$, from the exactness of the sequence (8) it follows that

$$
H^{n}\left(\mathfrak{g}, L\left(\lambda_{t_{\lambda}+1}\right)\right) \cong H^{n}\left(\mathfrak{g}, H^{0}\left(\lambda_{t_{\lambda}+1}\right)\right) \oplus H^{n-1}\left(\mathfrak{g}, H^{0}\left(\lambda_{t_{\lambda}+1}\right) / L\left(\lambda_{t_{\lambda}+1}\right)\right)
$$

Since $V(\lambda)$ is isomorphic to $H^{0}\left(-w_{0}(\lambda)\right)^{*}$, then, by Theorem $1.1 \mathrm{in} \mathrm{[30]} \mathrm{(pp.} \mathrm{3861-3862),}$ $H^{0}\left(\lambda_{t_{\lambda}+1}\right) / L\left(\lambda_{t_{\lambda}+1}\right) \cong L\left(\lambda_{t_{\lambda}}\right)$. Therefore,

$$
H^{n}\left(\mathfrak{g}, L\left(\lambda_{t_{\lambda}+1}\right)\right) \cong H^{n}\left(\mathfrak{g}, H^{0}\left(\lambda_{t_{\lambda}+1}\right)\right) \oplus H^{n-1}\left(\mathfrak{g}, L\left(\lambda_{t_{\lambda}}\right)\right) .
$$

According to the statement (a),

$$
H^{n}\left(\mathfrak{g}, L\left(\lambda_{t_{\lambda}}\right)\right) \cong\left\{\begin{array}{l}
0 \text { if } n<t_{\lambda} \\
k \text { if } n=t_{\lambda}
\end{array}\right.
$$

Then, by (10),

$$
H^{n}\left(\mathfrak{g}, L\left(\lambda_{t_{\lambda}+1}\right)\right) \cong\left\{\begin{array}{cc}
0 & \text { if } n<t_{\lambda}+1 \\
L\left(\alpha_{0}\right) & (1) \oplus k \text { if } n=t_{\lambda}+1
\end{array}\right.
$$

Now let $i \in\left\{t_{\lambda}+2, t_{\lambda}+3, \cdots, s\right\}$.

(E1) According to Formula (4), for all $i \in\left\{t_{\lambda}+2, t_{\lambda}+3, \cdots, s\right\}$,

$$
H^{n}\left(G_{1}, H^{0}\left(\lambda_{i}\right)\right) \cong\left\{\begin{array}{cc}
0 & \text { if } n<l\left(w_{i}\right) \\
L\left(\alpha_{0}\right)^{(1)} \text { if } n=l\left(w_{i}\right)
\end{array}\right.
$$

(E2) By the above (E1) and Formula (3), for all $i \in\left\{t_{\lambda}+2, t_{\lambda}+3, \cdots, s\right\}$,

$$
H^{n}\left(\mathfrak{g}, H^{0}\left(\lambda_{i}\right)\right) \cong\left\{\begin{array} { c c } 
{ 0 } & { \text { if } n < l ( w _ { i } ) , } \\
{ H ^ { n } ( G _ { 1 } , H ^ { 0 } ( \lambda _ { i } ) ) \text { if } n = l ( w _ { i } ) }
\end{array} \cong \left\{\begin{array}{cc}
0 & \text { if } n<l\left(w_{i}\right) \\
L\left(\alpha_{0}\right)^{(1)} \text { if } n=l\left(w_{i}\right)
\end{array}\right.\right.
$$

(E3) Consider the long cohomological exact sequence (8) for $i \in\left\{t_{\lambda}+2, t_{\lambda}+3, \cdots, s\right\}$. Since $H^{n}\left(\mathfrak{g}, H^{0}\left(\lambda_{i}\right)\right)=0$ for all $i \in\left\{t_{\lambda}+2, t_{\lambda}+3, \cdots, s\right\}$ and for all $n<l\left(w_{i}\right)$, from the exactness of the sequence (8) it follows that

$$
H^{n}\left(\mathfrak{g}, L\left(\lambda_{i}\right)\right) \cong H^{n}\left(\mathfrak{g}, H^{0}\left(\lambda_{i}\right)\right) \oplus H^{n-1}\left(\mathfrak{g}, H^{0}\left(\lambda_{i}\right) / L\left(\lambda_{i}\right)\right)
$$

for all $i \in\left\{t_{\lambda}+2, t_{\lambda}+3, \cdots, s\right\}$. By Theorem 1.1 in [30] (pp. 3861-3862),

$$
H^{0}\left(\lambda_{i}\right) / L\left(\lambda_{i}\right) \cong L\left(\lambda_{i-1}\right)
$$

for all $i \in\{1,2, \cdots, s\}$. Then, for all $i \in\left\{t_{\lambda}+2, t_{\lambda}+3, \cdots, s\right\}$,

$$
H^{n}\left(\mathfrak{g}, L\left(\lambda_{i}\right)\right) \cong H^{n}\left(\mathfrak{g}, H^{0}\left(\lambda_{i}\right)\right) \oplus H^{n-1}\left(\mathfrak{g}, L\left(\lambda_{i-1}\right)\right)
$$

Since $l\left(w_{i}\right) \leq t_{\lambda}$ for all $i \in\left\{t_{\lambda}+2, t_{\lambda}+3, \cdots, s\right\}$, by the statement $(a)$, it follows that $H^{n-1}\left(\mathfrak{g}, L\left(\lambda_{i-1}\right)\right)=0$ for all $i \in\left\{t_{\lambda}+2, t_{\lambda}+3, \cdots, s\right\}$ and for all $n<l\left(w_{i}\right)$. Then, by (11), for all $i \in\left\{t_{\lambda}+2, t_{\lambda}+3, \cdots, s\right\}$, 


$$
H^{n}\left(\mathfrak{g}, L\left(\lambda_{i}\right)\right) \cong H^{n}\left(\mathfrak{g}, H^{0}\left(\lambda_{i}\right)\right) \cong\left\{\begin{array}{cc}
0 & \text { if } n<l\left(w_{i}\right), \\
L\left(\alpha_{0}\right)^{(1)} & \text { if } n=l\left(w_{i}\right) .
\end{array}\right.
$$

In the case where $\mathfrak{g}=B_{l}, l\left(w_{i}\right)=2 l-i$. Therefore,

$$
H^{n}\left(\mathfrak{g}, L\left(\lambda_{i}\right)\right) \cong\left\{\begin{array}{cc}
0 & \text { if } n<2 l-i \\
L\left(\alpha_{0}\right)^{(1)} \text { if } n=2 l-i
\end{array}\right.
$$

Proof of Theorem 1 Statement (c). In this case $i=s=2 l-2$ and $l\left(w_{s}\right)=2 l-2$.

(F1) According to Formula (4),

$$
H^{n}\left(G_{1}, H^{0}\left(\lambda_{s}\right)\right) \cong\left\{\begin{array}{cc}
0 & \text { if } n<l\left(w_{s}\right), \\
L\left(\alpha_{0}\right)^{(1)} \text { if } n=l\left(w_{s}\right) .
\end{array}\right.
$$

(F2) By the above (F1) and Formula (3),

$$
H^{n}\left(\mathfrak{g}, H^{0}\left(\lambda_{s}\right)\right) \cong\left\{\begin{array} { c c } 
{ 0 } & { \text { if } n < l ( w _ { s } ) , } \\
{ H ^ { n } ( G _ { 1 } , H ^ { 0 } ( \lambda _ { s } ) ) } & { \text { if } n = l ( w _ { s } ) }
\end{array} \cong \left\{\begin{array}{cc}
0 & \text { if } n<l\left(w_{s}\right), \\
L\left(\alpha_{0}\right)^{(1)} \text { if } n=l\left(w_{s}\right) .
\end{array}\right.\right.
$$

(F3) Consider the long cohomological exact sequence (8) for $i=s$. Since $H^{n}\left(\mathfrak{g}, H^{0}\left(\lambda_{s}\right)\right)=0$ for all $n<l\left(w_{s}\right)$, from the exactness of the sequence (8) it follows that

$$
H^{n}\left(\mathfrak{g}, L\left(\lambda_{s}\right)\right) \cong H^{n}\left(\mathfrak{g}, H^{0}\left(\lambda_{s}\right)\right) \oplus H^{n-1}\left(\mathfrak{g}, H^{0}\left(\lambda_{s}\right) / L\left(\lambda_{s}\right)\right) .
$$

By Theorem 1.1 in [30] (pp. 3861-3862), $H^{0}\left(\lambda_{s}\right) / L\left(\lambda_{s}\right) \cong L\left(\lambda_{s-1}\right)$. Then,

$$
H^{n}\left(\mathfrak{g}, L\left(\lambda_{s}\right)\right) \cong H^{n}\left(\mathfrak{g}, H^{0}\left(\lambda_{s}\right)\right) \oplus H^{n-1}\left(\mathfrak{g}, L\left(\lambda_{s-1}\right)\right) .
$$

According to the statement (a),

$$
H^{n}\left(\mathfrak{g}, L\left(\lambda_{s-1}\right)\right) \cong\left\{\begin{array}{l}
0 \text { if } n<s-1 \\
k \text { if } n=s-1 .
\end{array}\right.
$$

Then, by (12),

$$
H^{n}\left(\mathfrak{g}, L\left(\lambda_{s}\right)\right) \cong\left\{\begin{array}{cc}
0 & \text { if } n<s \\
L\left(\alpha_{0}\right)^{(1)} \oplus k \text { if } n=s .
\end{array}\right.
$$

Proof of Theorem 2. We prove that, for all $j \in\{1,2, \cdots, s-1\}$, the dominant weight $\mu_{j}$ can be represented in the form $u_{j} \cdot 0+p \delta_{j}$ with $u_{j} \in W$ and $\delta_{j} \in X(T)^{+}$. For each of the root systems $R$ the description of the dominant weights $\mu_{1}, \mu_{2}, \cdots, \mu_{s-1}$ by the generators $s_{0}, s_{1}, \cdots, s_{l}$ of the affine Weyl group $W_{p}$ is given in Table 1. Using Table 1 and the expression for $s_{0}$ obtained in the proof of Theorem 1, we obtain for $u_{j}$ and $\delta_{j}$ the expressions that shown in Table 3. 
Table 3. Descriptions of $u_{j}$ and $\delta_{j}$.

\begin{tabular}{|c|c|c|c|}
\hline $\begin{array}{c}\text { Root } \\
\text { System }\end{array}$ & $j$ & $u_{j}$ & $\delta_{j}$ \\
\hline$A_{l}$ & $1,2, \cdots, s-1$ & $w_{1, l-2} w_{j, l}^{-1}$ & $\alpha_{0}$ \\
\hline$B_{l}$ & $\begin{array}{c}1,2, \cdots, l-1 \\
l, l+1, \cdots, s-1\end{array}$ & $\begin{array}{c}w_{1, l} w_{j+1, l-1}^{-1} w_{1, l} w_{1, l-1}^{-1} \\
w_{1,2 l-j-1} w_{1, l} w_{1, l-1}^{-1}\end{array}$ & $\omega_{2}$ \\
\hline$C_{l}$ & $\begin{array}{c}1,2, \cdots, l-2 \\
l-1, l, \cdots, s-2 \\
2 l-3\end{array}$ & $\begin{array}{c}w_{2, l} w_{3, l-1}^{-1} w_{1, l} w_{j+2, l}^{-1} \\
w_{2, l} w_{3, l-1}^{-1} w_{2,2 l-j-2} \\
w_{2, l} w_{2, l-1}^{-1}\end{array}$ & $\alpha_{0}$ \\
\hline$D_{l}$ & $1,2, \cdots, s-1$ & $w_{2, l-2} w_{3, l}^{-1} w_{1, l-2} w_{j+2, l}^{-1}$ & $\alpha_{0}$ \\
\hline
\end{tabular}

Since $l\left(w_{i, j}\right)=j-i+1$, using Table 3, we get

$$
l\left(u_{j}\right)=\left\{\begin{array}{l}
2 l-j-1 \text { if } g=A_{l}, \\
4 l-j-2 \text { if } g=B_{l}, \\
4 l-j-6 \text { if } g=C_{l}, \\
4 l-j-8 \text { if } g=D_{l}
\end{array}\right.
$$

for all $j \in\{1,2, \cdots, s-1\}$.

Proof of Theorem 2 Statement (a). We have

(G1) According to Formula (4), $H^{n}\left(G_{1}, H^{0}\left(\mu_{1}\right)\right)=0$ for all $n<l\left(u_{1}\right)$.

(G2) By the above (G1) and Formula (2), $H^{n}\left(\mathfrak{g}, H^{0}\left(\mu_{1}\right)\right)=0$ for all $n<l\left(u_{1}\right)$.

(G3) Consider the long cohomological exact sequence (5) for $\lambda=\mu_{1}$. Since $H^{n}\left(\mathfrak{g}, H^{0}\left(\mu_{1}\right)\right)=0$ for all $n<l\left(u_{1}\right)$, from this exact sequence it follows that

$$
H^{n}\left(\mathfrak{g}, L\left(\mu_{1}\right)\right) \cong H^{n-1}\left(\mathfrak{g}, H^{0}\left(\mu_{1}\right) / L\left(\mu_{1}\right)\right)
$$

for all $n<l\left(u_{1}\right)$. By Theorem 1.2 in [30] (p. 3862), $H^{0}\left(\mu_{1}\right) / L\left(\mu_{1}\right) \cong L\left(\lambda_{2}\right)$. Then,

$$
H^{n}\left(\mathfrak{g}, L\left(\mu_{1}\right)\right) \cong H^{n-1}\left(\mathfrak{g}, L\left(\lambda_{2}\right)\right)
$$

According to the statement (a) of Theorem 1,

$$
H^{n-1}\left(\mathfrak{g}, L\left(\lambda_{2}\right)\right) \cong\left\{\begin{aligned}
& 0 \text { if } n-1<2 \\
& k \text { if } n-1=2, \\
& H^{n-3}(\mathfrak{g}) \text { if } 2<n-1<l\left(w_{2}\right)
\end{aligned}\right.
$$

Therefore,

$$
H^{n}\left(\mathfrak{g}, L\left(\mu_{1}\right)\right) \cong\left\{\begin{aligned}
& 0 \text { if } n<3 \\
& k \text { if } n=3 \\
& H^{n-3}(\mathfrak{g}) \text { if } 3<n<l\left(w_{2}\right)+1
\end{aligned}\right.
$$

Proof of Theorem 2 Statement (b). We have

(H1) According to Formula (4), $H^{n}\left(G_{1}, H^{0}\left(\mu_{j}\right)\right)=0$ for all $j$ satisfying the condition $n<l\left(u_{j}\right)$.

(H2) By the above (H1) and Formula (2), $H^{n}\left(\mathfrak{g}, H^{0}\left(\mu_{j}\right)\right)=0$ for all $j$ satisfying the condition $n<l\left(u_{j}\right)$. 
(H3) Consider the long cohomological exact sequence (5) for $\lambda=\mu_{j}$ :

$$
H^{n}\left(\mathfrak{g}, L\left(\mu_{j}\right)\right) \rightarrow H^{n}\left(\mathfrak{g}, H^{0}\left(\mu_{j}\right)\right) \rightarrow H^{n}\left(\mathfrak{g}, H^{0}\left(\mu_{j}\right) / L\left(\mu_{j}\right)\right)
$$

Since $H^{n}\left(\mathfrak{g}, H^{0}\left(\mu_{i}\right)\right)=0$ for all $j$ satisfying the condition $n<l\left(u_{j}\right)$, from the exactness of the sequence (14) it follows that

$$
H^{n}\left(\mathfrak{g}, L\left(\mu_{j}\right)\right) \cong H^{n-1}\left(\mathfrak{g}, H^{0}\left(\mu_{j}\right) / L\left(\mu_{j}\right)\right)
$$

for all $j$ satisfying the condition $n<l\left(u_{j}\right)$. If $j=t_{\mu}+1$, then, by Formula (4),

$$
H^{t_{\mu}+1}\left(G_{1}, H^{0}\left(\mu_{t_{\mu}+1}\right)\right) \neq 0
$$

Hence, it follows that $j \leq t_{\mu}$.

By Lemma 4.1 in [30] (p. 3870), there exist the following short exact sequences:

$$
0 \rightarrow L\left(\mu_{1}\right) \oplus L\left(\lambda_{3}\right) \oplus L\left(\lambda_{1}\right) \rightarrow H^{0}\left(\mu_{2}\right) / L\left(\mu_{2}\right) \rightarrow L\left(\lambda_{2}\right) \rightarrow 0,
$$

and

$$
0 \rightarrow L\left(\mu_{j-1}\right) \oplus L\left(\lambda_{j+1}\right) \rightarrow H^{0}\left(\mu_{j}\right) / L\left(\mu_{j}\right) \rightarrow L\left(\lambda_{j}\right) \rightarrow 0
$$

for all $j \in\{3,4, \cdots, s-1\}$.

Further, we will use induction on $j$. If $j=2$, then, the long cohomological exact sequence corresponding to the short exact sequence (15) implies that

$0 \rightarrow H^{n-2}\left(\mathfrak{g}, L\left(\lambda_{2}\right)\right) \rightarrow H^{n-1}\left(\mathfrak{g}, L\left(\mu_{1}\right) \oplus L\left(\lambda_{3}\right) \oplus L\left(\lambda_{1}\right)\right) \rightarrow H^{n-1}\left(\mathfrak{g}, H^{0}\left(\mu_{2}\right) / L\left(\mu_{2}\right)\right) \rightarrow 0$ for all $n<l\left(u_{2}\right)$. Using the statement $(a)$ and Theorem 1 to the previous exact sequence, we obtain

$$
H^{n}\left(\mathfrak{g}, L\left(\mu_{2}\right)\right) \cong H^{n-1}\left(\mathfrak{g}, H^{0}\left(\mu_{2}\right) / L\left(\mu_{2}\right)\right) \cong\left\{\begin{array}{c}
0 \text { if } n<2 \text { and } n=3 \\
k \text { if } n=2,4 \\
H^{n-2}(\mathfrak{g}) \oplus H^{n-4}(\mathfrak{g}) \text { if } 4<n<l\left(u_{2}\right)
\end{array}\right.
$$

Therefore, the statement $(b)$ is valid for $j=2$.

Suppose the statement $(b)$ is valid for all $j<t$, where $t \leq t_{\mu}$. Then the long cohomological exact sequence corresponding to the short exact sequence (16) implies that

$$
0 \rightarrow H^{n-2}\left(\mathfrak{g}, L\left(\lambda_{t}\right)\right) \rightarrow H^{n-1}\left(\mathfrak{g}, L\left(\mu_{t-1}\right) \oplus L\left(\lambda_{t+1}\right)\right) \rightarrow H^{n-1}\left(\mathfrak{g}, H^{0}\left(\mu_{t}\right) / L\left(\mu_{t}\right)\right) \rightarrow 0
$$

for all $n<l\left(u_{t}\right)$. Using the statement $(a)$ and Theorem 1 to the previous exact sequence, we obtain $H^{n}\left(\mathfrak{g}, L\left(\mu_{t}\right)\right) \cong H^{n-1}\left(\mathfrak{g}, H^{0}\left(\mu_{t}\right) / L\left(\mu_{t}\right)\right) \cong\left\{\begin{array}{c}0 \text { if } n<t \text { and } n=t+1 \\ k \text { if } n=t, t+2 \\ H^{n-t}(\mathfrak{g}) \oplus H^{n-t-2}(\mathfrak{g}) \text { if } t+2<n<l\left(u_{t}\right)\end{array}\right.$

Therefore, the statement $(b)$ is valid for all $j \in\left\{2,3, \cdots, t_{\mu}\right\}$.

In the statement $(b)$, the case where $j=s-1$ for the Lie algebras of type $C_{l}$ is not covered. We consider this case in the statements (c).

Proof of Theorem 2 Statement (c). In this case $j=s-1=2 l-3$ and $l\left(\mu_{s-1}\right)=2 l-3$. 
(I1) According to Formula (4),

$$
H^{n}\left(G_{1}, H^{0}\left(\mu_{s-1}\right)\right) \cong\left\{\begin{array}{cc}
0 & \text { if } n<l\left(u_{s-1}\right) \\
L\left(\alpha_{0}\right)^{(1)} \text { if } n=l\left(u_{s-1}\right)
\end{array}\right.
$$

(I2) By the above (I1) and Formula (3),

$$
H^{n}\left(\mathfrak{g}, H^{0}\left(\mu_{s-1}\right)\right) \cong\left\{\begin{array} { c c } 
{ 0 \quad \text { if } n < l ( u _ { s - 1 } ) , } \\
{ H ^ { n } ( G _ { 1 } , H ^ { 0 } ( \mu _ { s - 1 } ) ) \text { if } n = l ( u _ { s - 1 } ) }
\end{array} \cong \left\{\begin{array}{cc}
0 & \text { if } n<l\left(u_{s-1}\right) \\
L\left(\alpha_{0}\right)^{(1)} \text { if } n=l\left(u_{s-1}\right)
\end{array}\right.\right.
$$

(I3) Consider the long cohomological exact sequence (14) for $j=s-1$. Since $H^{n}\left(\mathfrak{g}, H^{0}\left(\mu_{s-1}\right)\right)=0$ for all $n<l\left(u_{s-1}\right)$, from the exactness of the sequence (14) it follows that

$$
H^{n}\left(\mathfrak{g}, L\left(\mu_{s-1}\right)\right) \cong H^{n}\left(\mathfrak{g}, H^{0}\left(\mu_{s-1}\right)\right) \oplus H^{n-1}\left(\mathfrak{g}, H^{0}\left(\mu_{s-1}\right) / L\left(\mu_{s-1}\right)\right) .
$$

By Lemma 4.1 in [30] (p. 3870), there is the following exact sequence:

$$
0 \rightarrow L\left(\mu_{s-2}\right) \oplus L\left(\lambda_{s}\right) \rightarrow H^{0}\left(\mu_{s-1}\right) / L\left(\mu_{s-1}\right) \rightarrow L\left(\lambda_{s-1}\right) \rightarrow 0 .
$$

The long cohomological exact sequence corresponding to the short exact sequence (19) implies that

$$
0 \rightarrow H^{n-2}\left(\mathfrak{g}, L\left(\lambda_{s-1}\right)\right) \rightarrow H^{n-1}\left(\mathfrak{g}, L\left(\mu_{s-2}\right) \oplus L\left(\lambda_{s}\right)\right) \rightarrow H^{n-1}\left(\mathfrak{g}, H^{0}\left(\mu_{s-1}\right) / L\left(\mu_{s-1}\right)\right) \rightarrow 0 .
$$

Using the statement $(b)$ and Theorem 1 to the previous exact sequence, we get

$$
H^{n-1}\left(\mathfrak{g}, H^{0}\left(\mu_{s-1}\right) / L\left(\mu_{s-1}\right)\right) \cong\left\{\begin{array}{l}
0 \text { if } n<s-1 \\
k \text { if } n=s-1
\end{array}\right.
$$

Then, by (17), (18) and (20),

$$
H^{n}\left(\mathfrak{g}, L\left(\mu_{s-1}\right)\right) \cong\left\{\begin{array}{cc}
0 & \text { if } n<s-1 \\
L\left(\alpha_{0}\right) & (1) \oplus k \text { if } n=s-1
\end{array}\right.
$$

Proof of Corollary 3. By Theorem $1, H^{i}\left(\mathfrak{g}, L\left(\lambda_{i}\right)\right) \cong k$. Now, we show that $H^{i}\left(G_{1}, L\left(\lambda_{i}\right)\right) \cong k$. For all $i \in\left\{1,2, \cdots, t_{\lambda}\right\}$, there is the short exact sequence

$$
0 \rightarrow L\left(\lambda_{i}\right) \rightarrow H^{0}\left(\lambda_{i}\right) \rightarrow L\left(\lambda_{i-1}\right) \rightarrow 0
$$

By (4), $H^{i}\left(G_{1}, H^{0}\left(\lambda_{i}\right)\right)=0$ for all $i \in\left\{1,2, \cdots, t_{\lambda}\right\}$. Then, the long cohomological exact sequence of $G_{1}$-cohomology corresponding to the previous short exact sequence gives us the short exact sequence

$$
0 \rightarrow H^{i-1}\left(G_{1}, L\left(\lambda_{i-1}\right) \rightarrow H^{i}\left(G_{1}, L\left(\lambda_{i}\right)\right) \rightarrow 0\right.
$$

for all $i \in\left\{1,2, \cdots, t_{\lambda}\right\}$. Then, $H^{i}\left(G_{1}, L\left(\lambda_{i}\right)\right) \cong H^{i-1}\left(G_{1}, L\left(\lambda_{i-1}\right)\right.$ for all $i \in\left\{1,2, \cdots, t_{\lambda}\right\}$. If $i=1$, then $H^{1}\left(G_{1}, L\left(\lambda_{1}\right)\right) \cong H^{0}\left(G_{1}, L\left(\lambda_{0}\right)=H^{0}\left(G_{1}, L(0)\right) \cong k\right.$. Next, using induction on $i$ we obtain $H^{i}\left(G_{1}, L\left(\lambda_{1}\right)\right) \cong k$ for all $i \in\left\{1,2, \cdots, t_{\lambda}\right\}$. So, $H^{i}\left(\mathfrak{g}, L\left(\lambda_{i}\right)\right) \cong H^{i}\left(G_{1}, L\left(\lambda_{i}\right)\right) \cong k$ 
Now it suffices to show that $H^{i}\left(\mathfrak{g}, L\left(\lambda_{i}\right)\right) \cong H^{i}\left(G, L\left(\lambda_{i}\right)\right)$ for all $i \in\left\{1,2, \cdots, t_{\lambda}\right\}$. It is known that $H^{n}(G, V) \cong \operatorname{Hom}_{G}\left(k, H^{n}\left(G_{1}, V\right)^{(-1)}\right)$ for all simple modules with the highest restricted weight [38]. Then

$$
H^{i}\left(G, L\left(\lambda_{i}\right)\right) \cong \operatorname{Hom}_{G}\left(k, H^{i}\left(G_{1}, L\left(\lambda_{i}\right)\right)^{-1}\right) \cong k
$$

for all $i \in\left\{1,2, \cdots, t_{\lambda}\right\}$. Therefore, $H^{i}\left(\mathfrak{g}, L\left(\lambda_{i}\right)\right) \cong H^{i}\left(G, L\left(\lambda_{i}\right)\right)$ for all $i \in\left\{1,2, \cdots, t_{\lambda}\right\}$. $\square$.

\section{Discussion}

As noted in the Introduction, the results of this paper are part of a certain topical problem on cohomology of semisimple simply connected algebraic groups in positive characteristic and their Lie algebras. We were interested in studying the cohomology of simple modules for $\mathfrak{g}, G_{1}$, and $G$ in the restricted region, and the connections between them. In this paper, we have described the cohomology of a certain family of simple modules of the restricted region for $\mathfrak{g}$. These results can be used for further investigations of the connections between the cohomology of algebraic groups (cohomology for $G$ ) and both the usual cohomology (cohomology for $\mathfrak{g}$ ) and restricted cohomology (cohomology for $G_{1}$ ) of their Lie algebras. The results and the method of their proof can also be used to calculate the cohomology of simple modules for $G_{1}$ in the restricted region. Analyzing the results of Theorems 1 and 2, we can Formulate some statements, the validity of which is to be proved in further studies. For instance,

- If for all $i \in\left\{1,2, \cdots, t_{\lambda}\right\}$ and for all $n \leq i, H^{n}\left(\mathfrak{g}, L\left(\lambda_{i}\right)\right) \cong H^{n}\left(G, L\left(\lambda_{i}\right)\right)$.

- If for all $\mathrm{j} \in\left\{1,2, \cdots, t_{\mu}\right\}$ and for all $n \leq j, H^{n}\left(\mathfrak{g}, L\left(\mu_{i}\right)\right) \cong H^{n}\left(G, L\left(\mu_{i}\right)\right)$.

Similar research for small characteristics $(p \leq h)$ are also interesting open questions.

The results of this paper can also be used to calculate the cohomology of simple modules for Lie algebras related to classical Lie algebras.

Author Contributions: Conceptualization, S.S.I.; methodology, S.S.I.; software, L.S.K.; validation, S.S.I. and L.S.K.; formal analysis, S.S.I.; investigation, S.S.I., L.S.K. and S.K.M.; resources, S.K.M.; writing—original draft preparation, S.S.I. and L.S.K.; writing—review and editing, S.S.I. All authors have read and agreed to the published version of the manuscript.

Funding: This research was funded by the Science Committee of the Ministry of Education and Science of the Republic of Kazakhstan, grant number AP 08855935.

Institutional Review Board Statement: Not applicable.

Informed Consent Statement: Not applicable.

Data Availability Statement: No data were reported in the study.

Acknowledgments: The authors are grateful to the referees for valuable comments and suggestions that contributed to improving this paper.

Conflicts of Interest: The authors declare no conflict of interest.

\section{Appendix A}

We have mainly presented the results of this article in terms of cohomology for $\mathfrak{g}$ with coefficients in $k$. We offer the following information about the cohomology $H^{n}(\mathfrak{g})$ : Let

$$
H^{*}(\mathfrak{g})=\oplus_{n \geq 0} H^{n}(\mathfrak{g})
$$

and $p>h$, then, see [33] (p. 174), the following holds:

Theorem A1. Cohomology $H^{*}(\mathfrak{g})$ is an exterior algebra with 1 generators of dimensions

$$
2 d_{1}+1,2 d_{2}+1, \cdots, 2 d_{l}+1,
$$


where $d_{1}, d_{2}, \cdots, d_{l}$ are the exponents of $\mathfrak{g}$.

Here are the exponents of classical Lie algebras:

$d_{1}=1, d_{2}=2, \cdots, d_{l}=l$ for $\mathfrak{g}$ of type $A_{l}$

$d_{1}=1, d_{2}=3, \cdots, d_{l}=2 l-1$ for $\mathfrak{g}$ of type $B_{l}$ and $C_{l}$;

$d_{1}=1, d_{2}=3, \cdots, d_{l-1}=2 l-3, d_{l}=l-1$ for $\mathfrak{g}$ of type $D_{l}$;

$d_{1}=1, d_{2}=4, d_{3}=5, d_{4}=7, d_{5}=8, d_{6}=11$ for $\mathfrak{g}$ of type $E_{6}$;

$d_{1}=1, d_{2}=5, d_{3}=7, d_{4}=9, d_{5}=11, d_{6}=13, d_{7}=17$ for $\mathfrak{g}$ of type $E_{7} ;$

$d_{1}=1, d_{2}=7, d_{3}=11, d_{4}=13, d_{5}=17, d_{6}=19, d_{7}=23, d_{8}=29$ for $\mathfrak{g}$ of type $E_{8} ;$

$d_{1}=1, d_{2}=5, d_{3}=7, d_{4}=11$ for $\mathfrak{g}$ of type $F_{4}$;

$d_{1}=1, d_{2}=5$ for $\mathfrak{g}$ of type $G_{2}$.

Below we give some examples of the cohomology $H^{n}(\mathfrak{g})$ for the classical Lie algebras of rank 3.

Example A1. Let $p>3$ and $\mathfrak{g}$ be the classical simple Lie algebra of type $A_{3}$.Then

$$
H^{n}(\mathfrak{g}) \cong\left\{\begin{array}{c}
k \text { if } n=0,3,5,7,8,10,12,15 ; \\
0 \text { otherwise }
\end{array}\right.
$$

Proof. The exponents of the Lie algebra $\mathfrak{g}$ are $d_{1}=1, d_{2}=2, d_{3}=3$. By Theorem 1 , the generators of the cohomology $H^{*}(\mathfrak{g})$ as an exterior algebra are the classes of cocycles of degrees 3,5 , and 7. They span the cohomology $H^{3}(\mathfrak{g}), H^{5}(\mathfrak{g})$, and $H^{7}(\mathfrak{g})$, respectively. Obviously, $H^{1}(\mathfrak{g})=0$ and $H^{2}(\mathfrak{g})=0$. Then, we get $H^{0}(\mathfrak{g})=\wedge^{0} H^{3}(\mathfrak{g}) \neq 0$. Since, $H^{i}(\mathfrak{g}) \wedge$ $H^{i}(\mathfrak{g})$ for all $i>0$ and $\operatorname{dimg}=15$, then, for all $n>2$ the following holds:

$$
H^{n}(\mathfrak{g})=\sum_{i+j=n} H^{i}(\mathfrak{g}) \wedge H^{j}(\mathfrak{g})+\sum_{i+j+t=n} H^{i}(\mathfrak{g}) \wedge H^{j}(\mathfrak{g}) \wedge H^{t}(\mathfrak{g}) .
$$

The last formula yields $H^{8}(\mathfrak{g})=H^{3}(\mathfrak{g}) \wedge H^{5}(\mathfrak{g}) \neq 0, H^{10}(\mathfrak{g})=H^{3}(\mathfrak{g}) \wedge H^{7}(\mathfrak{g}) \neq 0$, $H^{12}(\mathfrak{g})=H^{5}(\mathfrak{g}) \wedge H^{7}(\mathfrak{g}) \neq 0, H^{15}(\mathfrak{g})=H^{3}(\mathfrak{g}) \wedge H^{7}(\mathfrak{g}) \wedge H^{7}(\mathfrak{g}) \neq 0$; otherwise, $H^{n}(\mathfrak{g})=0$. Since the dimension of the exterior algebra with $l$ generators is equal to $2^{l}$, then all nontrivial cohomology has dimension 1.

Calculations similar to Example A1 give us:

Example A2. Let $p>5$ and $\mathfrak{g}$ be the classical simple Lie algebra of type $B_{3}$ or $C_{3}$. Then

$$
H^{n}(\mathfrak{g}) \cong\left\{\begin{array}{c}
k \text { if } n=0,3,7,10,11,14,18,21 ; \\
0 \text { otherwise }
\end{array}\right.
$$

\section{References}

1. Cartan, É. Sur les invariants intégraux de certains espaces homogènes clos. Ann. Soc. Pol. Math. 1929, 8, 181-225.

2. Chevalley, C.; Eilenberg, S. Cohomology theory of Lie groups and Lie algebras. Trans. Am. Math. Soc. 1948, 63, 85-124. [CrossRef]

3. Hochschild, G. Cohomology of restricted Lie algebras. Am. J. Math. 1954, 76, 555-580. [CrossRef]

4. Greub, W.; Halperin, S.; Vanstone, R. Connections, Curvature and Cohomology. Cohomology of Principal Bundles and Homogeneous Spaces; 3; Academic Press: Cambridge, MA, USA, 1975.

5. Weyl, H. The Theory of Groups and Quantum Mechanics; Dover Publications: New York, NY, USA, 1931; pp. 175-180, $210-217$.

6. Howe, R. On the role of the Heisenberg group in harmonic analysis. Bull. Am. Math. Soc. 1980, 3, 821-844. [CrossRef]

7. Belavin, A.A.; Polyakov, A.M.; Zamolodchikov, A.B. Infinite conformal symmetry in two-dimensional quantum field theory. Nucl. Phys. B 1984, 241, 333-380. [CrossRef]

8. Andrzejewski, T.; Figueroa-O'Farrill, J.M. Kinematical Lie algebras in 2+1 dimensions. J. Math. Phys. 2018, 59, 061703. [CrossRef]

9. Figueroa-O'Farrill, J.M. Kinematical Lie algebras via deformation theory. J. Math. Phys. 2018, 59, 061701. [CrossRef]

10. Truini, P.; Marrani, A.; Rios, M.; Irwin, K. Space, Matter and Interactions in a Quantum Early Universe Part I: Kac-Moody and Borcherds Algebras. Symmetry 2021, 13, 2342. [CrossRef] 
11. Magazev, A.A.; Boldyreva, M.N. Schrödinger Equations in Electromagnetic Fields: Symmetries and Noncommutative Integration. Symmetry 2021, 13, 1527. [CrossRef]

12. Sun, Q.; Wu, Z. Cohomologies of n-Lie Algebras with Derivations. Mathematics 2021, 9, 2452. [CrossRef]

13. Alvarez, M.A.; Rosales-Gómez, J. Cohomology of Lie Superalgebras. Symmetry 2020, 12, 833. [CrossRef]

14. Ludkowski, S.V. Cohomology Theory of Nonassociative Algebras with Metagroup Relations. Axioms 2019, 8, 78. [CrossRef]

15. Ludkowski, S.V. Separability of Nonassociative Algebras with Metagroup Relations. Axioms 2019, 8, 139. [CrossRef]

16. Ludkowski, S.V. Homotopism of Homological Complexes over Nonassociative Algebras with Metagroup Relations. Mathematics 2021, 9, 734. [CrossRef]

17. Ludkowski, S.V. Torsion for Homological Complexes of Nonassociative Algebras with Metagroup Relations. Axioms 2021, 10, 319. [CrossRef]

18. Friedlander, E.M.; Parshall, B.J. Modular representation theory of Lie algebras. Am. J. Math. 1988, 110, 1055-1093. [CrossRef]

19. Farnsteiner, F. Cohomology groups of restricted enveloping algebras. Math. Z. 1991, 206, 103-117. [CrossRef]

20. Jantzen, J.C. First cohomology groups for classical Lie algebras. Prog. Math. 1991, 95, 291-315.

21. Dzhumadil'daev, A.S. On the cohomology of modular Lie algebras. Math. USSR-Sb. 1984, 47, 127-143. [CrossRef]

22. Dzhumadil'daev, A.S.; Ibraev, S.S. Nonsplit extensions of modular Lie algebras of rank 2. Homol. Homotopy Appl. 2002, 4, 141-163. [CrossRef]

23. Ibraev, S.S. Cohomology of $\mathfrak{s l}_{3}$ and $\mathfrak{g l}_{3}$ with coefficients in simple modules and Weyl modules in positive characteristics. Symmetry Integr. Geom. Methods Appl. 2021, in press.

24. Ibraev, S.S.; Turbayev, B.E. Cohomology for the Lie algebra of type $A_{2}$ over a field of characteristic 2. Sib. Èlectron. Mat. Izv. 2021, 18, 729-739. [CrossRef]

25. Ibrayeva, A.A.; Ibraev, S.S.; Yeshmurat, G.K. Cohomology of simple modules for $\mathfrak{s l}_{3}(k)$ in characteristic 3 . Bull. Karaganda Univ. Math. Ser. 2021, 3, 36-43. Available online: https://mathematics-vestnik.ksu.kz/apart/2021-103-3/4.pdf (accessed on 1 December 2021). [CrossRef]

26. Rudakov, A.N. Deformations of simple Lie algebras. Math. USSR-Izv. 1971, 5, 1120-1126. [CrossRef]

27. Kuznetsov, M.I.; Chebochko, N.G. Deformations of classical Lie algebras. Sb. Math. 2000, 191, 1171-1190. Available online: https:/ / ui.adsabs.harvard.edu/link_gateway/2000SbMat.191.1171K/doi:10.1070/SM2000v191n08ABEH000499 (accessed on 1 December 2021). [CrossRef]

28. Chebochko, N.G. Deformations of classical Lie algebras with homogeneous root system in characteristic two. I. Sb. Math. 2005, 196, 1371-1402. [CrossRef]

29. O'Halloran, J. Weyl modules and the cohomology of Chevalley groups. Am. J. Math. 1981, 103, 399-410. [CrossRef]

30. Ibraev, S.S. Some Weyl modules and cohomology for algebraic groups. Commun. Algebra 2020, 48, 3859-3873. [CrossRef]

31. Ibraev, S.S. On the first cohomology of an algebraic group and its Lie algebra in positive characteristic. Math. Notes 2014, 96, 491-498. [CrossRef]

32. Ibraev, S.S. On the second cohomology of an algebraic group and of its Lie algebra in a positive characteristic. Math. Notes 2017, 101, 841-849. [CrossRef]

33. Chiu, S.; Shen, G. Cohomology of Cartan type Lie algebras of characteristic p. Abh. Math. Sem. Univ. Humburg 1986, 57, 139-156.

34. Feigin, B.L.; Fuchs, D.B. Cohomologies of Lie groups and Lie algebras. Itogi Nauli i Tekh. Ser. Sovrem. Probl. Mat. Fundam. Napravleniya 1988, 21, 121-209.

35. Jantzen, J.C. Representations of Algebraic Groups, 2nd ed.; Mathematical Surveys and Monographs; American Mathematical Society: Providence, RI, USA, 2003; Volume 107.

36. Feldvoss, J. Homological topics in the representation theories of restricted Lie algebras. Contemp. Math. 1996, 194, 69-120.

37. Andersen, H.H.; Jantzen, J.C. Cohomology of induced representations for algebraic groups. Math. Ann. 1984, 269, 487-524. Available online: http:/ / eudml.org/doc/163945 (accessed on 1 December 2021). [CrossRef]

38. Ibraev, S.S.; Kainbaeva, L.S.; Menlikozhayeva, S.K. Cohomology of simple modules for algebraic groups. Bull. Karaganda Univ. Math. Ser. 2020, 1, 37-43. Available online: https://mathematics-vestnik.ksu.kz/apart/2020-97-1/4.pdf (accessed on 1 December 2021). [CrossRef] 